\title{
Afasia global sem hemiparesia: AVC ou transtorno conversivo?
}

\section{Global aphasia without hemiparesis: stroke or conversion disorder?}

\author{
Daniel Philippi de Negreiros ${ }^{1}$, Felipe Fregni², Andréia Zavaloni Scalco ${ }^{3}$ \\ ${ }^{1}$ Médico psiquiatra. \\ 2 Instrutor de Neurologia da Harvard Medical School.
}

${ }^{3}$ Médica-assistente do Instituto de Psiquiatria do Hospital das Clínicas da Faculdade de Medicina da Universidade de São Paulo (IPqHC-FMUSP), Clinical Research Fellow da University of Toronto.

Recebido: 25/02/2006 - Aceito: 29/05/2006

\section{Resumo}

Contexto: A realização de diagnóstico neurológico e psiquiátrico em ambiente de emergência hospitalar com frequiência é uma tarefa complexa e exige colaboração interdisciplinar. Um dos diagnósticos diferenciais de doenças neurológicas é o transtorno conversivo, cuja característica principal é a presença de sintomas afetando funções motoras ou sensoriais, que sugerem desordem clínica ou neurológica, porém sem doença orgânica subjacente que explique o quadro. Relato de caso: Os autores relatam o caso de uma paciente de 23 anos com apresentação clínica atípica de acidente vascular cerebral, afasia global sem hemiparesia, que foi inicialmente diagnosticada como transtorno psiquiátrico pelo serviço de clínica médica de emergência. Conclusão: Certas apresentações neurológicas podem ser interpretadas como transtorno conversivo pelas similaridades clínicas entre as duas desordens, raridade do quadro clínico, pela presença de sintomatologia psiquiátrica e fatores psicossociais nos pacientes neurológicos. Mesmo com apresentações neurológicas atípicas e sintomas psiquiátricos, pacientes com quadro sugestivo de transtorno conversivo devem ser sempre investigados de forma interdisciplinar.

Negreiros, D.P. et al. / Rev. Psiq. Clín. 34 (1); 23-27, 2007

Palavras-chave: Acidente cerebrovascular, transtorno conversivo, afasia, diagnóstico diferencial, serviços médicos de emergência.

\section{Abstract}

Background: The neurologic and psychiatric diagnosis in emergency settings are difficult tasks and require interdisciplinary effort. Conversion disorder is one of the differential diagnosis for certain neurologic disorders. The main characteristic is motor or sensory deficits suggesting neurologic or medical condition, but without organic disease that explains the symptoms. Case report: We present a 23 year-old-woman with an atypical clinical presentation of stroke: global aphasia without hemiparesis. This patient was initially diagnosed with conversion disorder by the internal medicine service in the emergency room. Conclusion: Some rare neurologic diseases can be interpreted as conversive disorders due to some reasons: clinical similarities between the two disorders, unusual clinical presentations, comorbidity of neurologic and psychiatric disorders and psychological features of the neurological patients. Patients presenting with atypical neurological or psychiatric symptoms, in which a diagnosis of conversion disorder is suspected, should always be investigated in an interdisciplinary setting.

Negreiros, D.P. et al. / Rev. Psiq. Clín. 34 (1); 23-27, 2007

Key-words: cerebrovascular accident, conversion disorder, aphasia, diagnosis differential, emergency medical services. 


\section{Introdução}

A realização de diagnóstico neurológico e psiquiátrico em ambiente de emergência hospitalar com frequiência é uma tarefa complexa e exige colaboração interdisciplinar.

Um dos diagnósticos diferenciais psiquiátricos de doenças neurológicas é o transtorno conversivo (TC), caracterizado pela presença de sinais e sintomas afetando funções motoras ou sensoriais, que sugerem desordem clínica ou neurológica, porém sem doença orgânica subjacente que explique o quadro. $\mathrm{O}$ diagnóstico de TC requer ainda a existência de fatores psicológicos associados ao início ou exacerbação dos sintomas (APA, 1994).

Apesar de não existirem dados estatísticos confiáveis acerca da frequiência de casos de TC em serviços de emergência médica, estima-se que a sua ocorrência seja elevada (Glick et al., 2000). Nas interconsultas psiquiátricas em hospital geral, TC é o transtorno somatoforme mais freqüente, diagnosticado em $5 \%$ a $16 \%$ dos casos consultados (Ford e Parker, 1991; Guggenheim e Smith, 1995; Smaira et al., 2003).

A análise da literatura sugere que a realização de diagnóstico errôneo de TC era mais comum no passado. Slater e Glithero (1965) relataram a realização de novos diagnósticos em quase metade dos casos inicialmente diagnosticados como "histeria". Todavia, estudos mais recentes demonstram que a taxa de re-diagnóstico diminuiu muito, atribuindo tal mudança ao desenvolvimento de métodos diagnósticos mais apurados (Mace e Trimble, 1996; Binzer e Kullgren, 1998; Crimlisk et al., 1998). Outra explicação para a redução de erros diagnósticos observada após a década de 1970 é a melhoria na qualidade desses estudos, já que os anteriores apresentavam importantes falhas metodológicas (Stone et al., 2005).

Tanto o diagnóstico errôneo de TC quanto o excesso de exames utilizados para descartá-lo são potencialmente prejudiciais, seja pelo atraso no tratamento da condição clínica ou psiquiátrica subjacente, seja pelo uso ineficiente dos recursos diagnósticos disponíveis (Stone et al., 2005).

Neste artigo descrevemos o caso de uma paciente avaliada em pronto-socorro com quadro de afasia, dando ênfase à dificuldade do diagnóstico diferencial entre AVC e TC e à importância da avaliação multidisciplinar.

\section{Relato de caso}

A., 23 anos, casada, do lar, $2^{\circ}$ grau completo, evangélica. Foi trazida pelo marido e pela irmã ao Serviço de
Emergência por não conseguir falar. Os acompanhantes relatavam que a paciente, após voltar de um culto, chegou a sua casa sem falar, expressando-se apenas por sons incompreensíveis e gestos. Foi levada nos dois dias seguintes a dois serviços de pronto-atendimento, recebendo o diagnóstico de amigdalite e "nervoso". Nas semanas anteriores, A. vinha desconfiando de uma possível traição do marido, o que havia motivado desentendimentos entre o casal. Na noite anterior ao início dos sintomas, o casal teve nova discussão e a paciente foi morar com a irmã, relatando estar muito angustiada. Não havia antecedentes mórbidos nem quadro prévio de sintomas neurológicos. A. nunca havia realizado tratamento psiquiátrico, porém a família relatava uma tentativa de suicídio no passado. Apesar de ser considerada uma pessoa "nervosa" pelos familiares, não foi possível estabelecer nenhum diagnóstico psiquiátrico prévio. A família negava o aparecimento de outros possíveis déficits neurológicos transitórios, como o acometimento da função motora. Após ser avaliada pelo serviço de clínica médica, foi solicitada interconsulta psiquiátrica, com a hipótese diagnóstica de "síndrome conversiva".

A. estava acompanhada de familiares, com vestes próprias e adequadas para a situação. Encontrava-se consciente, porém incapaz de se expressar verbalmente, emitindo apenas sons incompreensíveis. Tampouco foi capaz de escrever ou obedecer a comandos verbais. Mostrava-se calma, sem polarização do humor e sem sinais de ansiedade. Não era possível avaliar o pensamento, a memória, a inteligência, o juízo ou a capacidade crítica. Não foram observados outras alterações psicomotoras nem indícios de alterações sensoperceptivas.

A aparente afasia de compreensão observada no exame psíquico gerava dúvidas quanto à etiologia do quadro, o que levou à solicitação de avaliação neurológica.

No exame neurológico, a paciente apresentava-se com atenção preservada. Apenas emitia sons, não sendo capaz de pronunciar uma única palavra, ler, escrever, repetir nem nomear. A compreensão também estava prejudicada: quando questionada se o examinador estava usando uma camisa de uma determinada cor, ela sempre concordava balançando a cabeça, independentemente da cor questionada. A força motora era simétrica e grau $\mathrm{V}$ nos quatro membros, com reflexos simétricos e cutâneo plantar em flexão bilateralmente. Não havia déficits de sensibilidade nem do campo visual. Apesar de haver dúvidas quanto ao diagnóstico de afasia global (AG) em razão da ausência de déficits motores, foi solicitado exame de neuroimagem. 
A tomografia computadorizada de crânio revelou uma área sugestiva de infarto em regiões temporal, occipital e parietal posterior à esquerda (Figura 1). A paciente foi internada na enfermaria de neurologia para investigação etiológica do acidente vascular cerebral isquêmico (AVCi). A ressonância magnética de encéfalo mostrou uma área isquêmica parietal extensa com um pequeno acometimento frontal posterior subcortical à esquerda, acometendo território de artéria cerebral média (ACM) esquerda. A angiografia cerebral revelou características de embolia e obstrução de ramos distais da artéria cerebral média esquerda (Figura 2). Exames subseqüentes para investigação etiológica de AVC isquêmico (AVCi) em jovem, seguindo o protocolo da clínica neurológica do HC-FMUSP (incluindo a investigação de doenças trombofílicas e vasculites), não revelaram nenhuma anormalidade, sendo diagnosticado AVC de etiologia indeterminada (Adams et al., 1993).

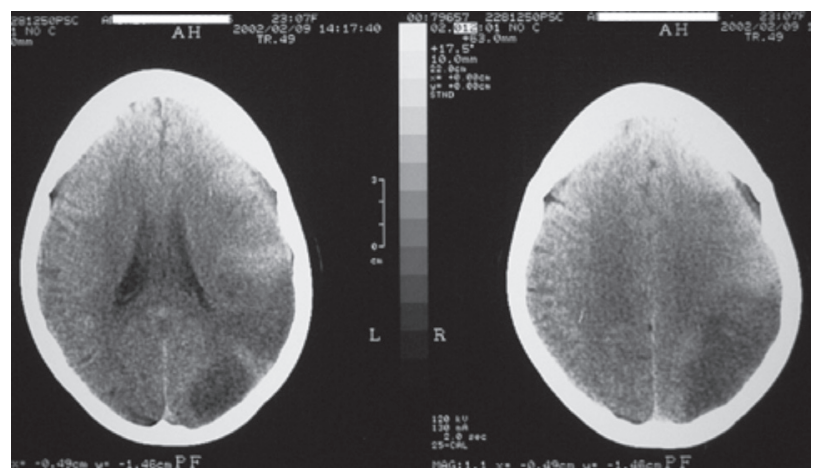

Figura 1. Tomografia computadorizada de crânio realizada na admissão, mostrando lesão hipoatenuante em áreas parietal, temporal e frontal à esquerda, sugestiva de quadro isquêmico vascular em área de artéria cerebral média.

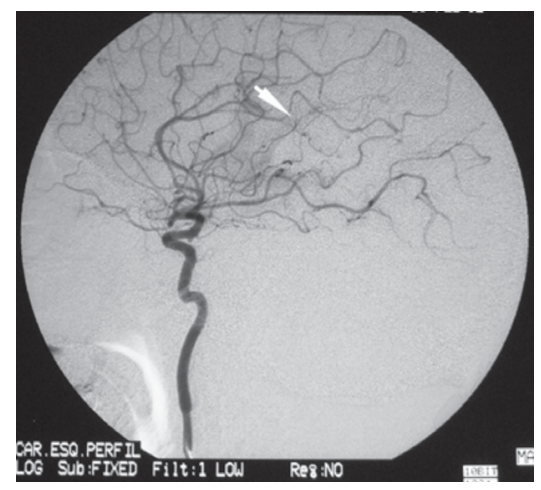

Figura 2. Angiografia cerebral revelando características de embolia e obstrução de ramos distais da artéria cerebral média esquerda.

\section{Discussão}

Uma das principais dificuldades no raciocínio clínico desse caso está relacionada à raridade do quadro clínico da paciente (AG sem hemiparesia). Apesar de existirem casos relatados de AG sem hemiparesia, esse diagnóstico é sempre difícil de ser realizado. A AG é geralmente causada por oclusão proximal da artéria cerebral média $(\mathrm{ACM})$ ou da artéria carótida interna (ACI). Nesses casos, no entanto, ocorre um infarto do giro pré-central (área motora primária), ocasionando um quadro de hemiparesia de predomínio braquiofacial (Tranel et al., 1987). Poucos casos foram relatados na literatura de pacientes com AG sem hemiparesia, sendo explicados por lesões confinadas à área pré-rolândica de linguagem ou, então, como resultado de duas lesões discretas afetando áreas de linguagem posterior e anterior e poupando o giro pré-central (Legatt et al., 1987; Deleval et al., 1989). No caso descrito, entretanto, a lesão era restrita à porção posterior do giro pré-central, afetando uma área classicamente atribuída à compreensão da linguagem. Algumas hipóteses poderiam explicar a dissociação clínico-anatômica vista no presente estudo: 1) A lesão apresentava um pequeno acometimento frontal que poderia estar afetando vias de condução e ocasionando o déficit de fluência (Bang et al., 2004). 2) A paciente apresentou uma lesão frontal transitória que afetou territórios de linguagem anteriores e tal lesão, apesar de reversível, poderia apresentar efeito funcional prolongado. Entretanto, a evolução lenta da paciente contraria tal hipótese. Outro fator que pode trazer dificuldade diagnóstica nesses pacientes com AG sem hemiparesia é a preservação da atenção, visto que lesões que resultam em AG geralmente são extensas, causando déficit nessa função cognitiva.

É possível também que um estudo neuropsicológico detalhado, tal como investigação de alterações visuoespaciais, construcionais, praxias, gnosias, entre outras, daria pistas importante sobre o comprometimento focal de certas funções cognitivas e, que certamente, facilitaria o diagnóstico de AVCi. Porém, tal exame neuropsicológico não é factível de ser realizado em um ambiente de emergência em virtude do tempo para a sua realização (1 a 3 horas) e da necessidade de neuropsicólogo treinado.

O bom estado geral, a atenção preservada, a ausência de sinais motores evidentes, a idade jovem, a ausência de fatores de risco importantes para doenças vasculares (exceto o uso de anticoncepcional), associados a características clínicas e epidemiológicas para TC, como ser 
do sexo feminino, jovem, possuir apresentação neurológica atípica e clara situação de dificuldade psicológica associada temporalmente aos sintomas, devem ter sido potenciais confundidores no raciocínio diagnóstico da paciente no setor de emergência. Teasell e Shapiro (2002) sugeriram que os principais fatores associados ao diagnóstico de TC, quando, na verdade, há alguma condição neurológica que explique os sintomas, são: 1) história de dificuldades psicológicas; 2) apresentação neurológica não usual; 3) testes diagnósticos normais; e 4) sexo feminino. Tal observação ressalta a importância do diagnóstico diferencial, haja vista a grande semelhança desses fatores com algumas características epidemiológicas e critérios diagnósticos do TC (Steinhart, 1980; APA, 1994; Guggenheim e Smith, 1995).

Por outro lado, há fatores clínicos e de história que podem contribuir para o diagnóstico de TC. Em particular, a presença de sintomas múltiplos, sintomas depressivos e/ou ansiosos, história prévia de sintomas conversivos ou de cirurgias sem achados positivos aumentam a possibilidade de que o sintoma primário seja conversivo (Stone et al., 2002). Também já foram associados ao TC: possuir procedência de área rural, história familiar de TC e história de abuso físico e/ou sexual na infância (Roelofs et al., 2002).

Uma história de dificuldades psicológicas deve ser cuidadosamente investigada, com especial atenção à conexão temporal entre a sua ocorrência e o desenvolvimento ou agravamento dos sintomas (Guggenheim e Smith, 1995). Todavia, apesar de fundamental para o diagnóstico de TC, esse dado não deve ser supervalorizado, já que os conflitos psicológicos também são freqüentes em pacientes com doenças neurológicas (Ewald et al., 1994; Lishman, 1998), como pôde ser observado neste relato.

No presente caso, outro aspecto que também pode ter contribuído para a hipótese diagnóstica inicial de TC foi a indiferença afetiva observada na paciente perante seus sintomas. Tal característica, denominada la belle indifference, costumava ser associada ao TC (Steinhart, 1980; Freeman, 1992). Esse termo, inicialmente descrito por Freud, ganhou popularidade em meados do século de 1920 na literatura psicoanalítica. A interpretação clássica da indiferença afetiva se baseia no ganho primário, ou seja, na conversão de um conflito inconsciente em um sintoma físico. No entanto, essa hipótese contrasta com as altas taxas de sintomas depressivos e ansiosos presentes em pacientes com TC (Stone et al., 2006).
Além do mais, é possível que pacientes afásicos em estágios iniciais não apresentem a preocupação esperada com o problema, por estarem incapacitados de monitorar seus próprios déficits numa perspectiva apropriada (Benson, 1992). Inclusive, em pacientes com afasia de Broca, a ausência de preocupação com o déficit pode ser indicativa de dano cerebral mais extenso (Lishman, 1987). Em uma recente revisão sistemática, Stone et al. (2006) afirmaram que la belle indifference deve ser abandonada como característica clínica do TC, em virtude da sua freqüente associação com doenças orgânicas.

Apesar de a afasia histérica já ter sido descrita na literatura (Levy e Jankovic, 1983), a manifestação de linguagem mais comum no TC é afonia ou a produção de sons incompreensíveis (Lishman, 1987; Guggenheim e Smith, 1995), o que ressalta a baixa precisão da presença de afasia para o diagnóstico de TC.

Em casos de suspeita de TC, testes diagnósticos normais também devem ser interpretados com cautela (Stoudemire et al., 1982). Em ambientes de emergência médica, a disponibilidade de testes diagnósticos específicos para condições clínicas raras não costuma ser freqüente. Ademais, erros laboratoriais, de interpretação diagnóstica ou, ainda, a inexistência de testes específicos podem justificar os achados negativos (Trojano e Paolicelli, 2001; Roach eSparagana, 2004). Mace e Trimble (1996) citaram o exemplo de uma mulher com extrema demanda de atenção que não teve sua tomografia tão bem avaliada, deixandose de diagnosticar a verdadeira causa neurológica, o que ilustra o risco de um diagnóstico errôneo de TC.

É importante ressaltar que, mesmo diante dos casos positivos para TC, a sua presença não irá impedir os pacientes de desenvolverem doenças graves (Crimlisk et al., 1998) e que não se deve descartar a hipótese de re-diagnóstico, atualmente estimada em 4\% (Stone et al., 2005).

Sendo assim, a investigação inicial feita de maneira criteriosa e a avaliação psiquiátrica precoce tornam o diagnóstico de TC mais confiável (Mace e Trimble, 1996; Binzer e Kullgren, 1998). Todavia, a avaliação psiquiátrica nem sempre é realizada. No estudo de Slater e Glithero (1965), um dos mais citados acerca da questão do diagnóstico errôneo de TC, menos de um terço dos pacientes que receberam o diagnóstico de "histeria" foi avaliado por psiquiatra. Binzer e Kullgren (1998), num estudo com até cinco anos de seguimento, observaram que nenhum paciente diagnosticado como TC havia sido referido inicialmente ao psiquiatra e que apenas $10 \%$ consultaram psiquiatras no decorrer do estudo. 
Por outro lado, pacientes com TC são muitas vezes submetidos a um grande número de testes diagnósticos desnecessários em razão da falta de um diagnóstico acurado (Dula e Denaples, 1995; Mace e Trimble, 1996; Crimlisk et al., 1998).

Uma história cuidadosa é essencial (Stone et al., 2002). Avaliar os sintomas clínicos característicos, examinar aspectos psicológicos subjacentes e estabelecer uma cooperação entre psiquiatras e clínicos de outras especialidades precocemente pode ajudar a evitar a progressão para cronicidade em pacientes com TC (Leslie, 1988; Ophir et al., 1990; Leo e Konakanchi, 1999).

Ao elevar a confiabilidade do diagnóstico de TC, a participação do psiquiatra poderá contribuir para a racionalização do uso de recursos diagnósticos e, mediante a sua participação em equipes multidisciplinares, prevenir condutas clínicas menos criteriosas diante de pacientes que apresentem dificuldades psicológicas ou outras características clínicas sugestivas de TC.

\section{Conclusão}

O diagnóstico diferencial entre doenças neurológicas e TC costuma ser difícil, em virtude, entre outros fatores, das similaridades clínicas entre as duas desordens e da freqüiente presença de sintomatologia psiquiátrica nos pacientes neurológicos. Assim, anamnese detalhada, exames físicos geral e neurológico, exames complementares e avaliação psiquiátrica são necessários no diagnóstico e manejo desses pacientes, reduzindo o risco de erro diagnóstico e a utilização ineficiente dos serviços de saúde.

\section{Referências}

Adams, H. P. J.; Bendixen, B. H.; Kappelle, L. J.; Biller, J.; Love, B. B.; Gordon, D. L.; Marsh, E. E. R. Classification of subtype of acute ischemic stroke. Definitions for use in a multicenter clinical trial. Toast. Trial of org 10172 in acute stroke treatment. Stroke 24(1): 35-41, 1993.

APA - American Psychiatric Association. Diagnostic and statistical manual of mental Disorders: DSM-IV. American Psychiatric Association, Washington, DC, p. 886, 1994.

Bang, O. Y.; Heo, K. G.; Kwak, Y.; Lee, P. H.; Joo, I. S.; Huh, K. Global aphasia without hemiparesis: lesion analysis and its mechanism in 11 Korean patients. J Neurol Sci 217(1): 101-6, 2004.

Benson, D. Neuropsychiatric aspects of aphasia and related language impairments. In: Yudofski, S. (Ed.) Textbook of neuropsychiatry. The American Psychiatry Press, Washington DC, pp. 311-327, 1992.

Binzer, M.; Kullgren, G. Motor conversion disorder. A prospective 2- to 5-year follow-up study. Psychosomatics 39(6): 519-27, 1998.

Crimlisk, H. L.; Bhatia, K.; Cope, H.; David, A.; Marsden, C. D.; Ron, M. A. Slater revisited: 6 year follow up study of patients with medically unexplained motor symptoms. BMJ 316(7131): 582-6, 1998.

Deleval, J.; Leonard, A.; Mavroudakis, N.; Rodesch, G. Global aphasia withouthemiparesis following prerolandic infarction. Neurology 39(11): 1532-5, 1989.
Dula, D. J.; Denaples, L. Emergency department presentation of patients with conversion disorder. Acad Emerg Med 2(2): 120-3, 1995.

Ewald, H.; Rogne, T.; Ewald, K.; Fink, P. Somatization In patients newly admitted to a neurological department. Acta Psychiatr Scand 89(3): 174-9, 1994

Ford, C. V.; Parker, P. E. Somatization in consultation-liaison psyhiatry. In: Kirmayer, L. J.; Robbins, J. M. (eds.). Current concepts of somatization: research and clinical perspectives. The American Psychiatry Press, Washington DC, pp. 143-155, 1991.

Freeman, C. Neurotic disorders, In: Kendell, R.; Zealley, A. (eds.). Companion to Psychiatric Studies. Churchill Livingstone, Edinburgh, pp. 485-525, 1992.

Glick, T. H.; Workman, T. P.; Gaufberg, S. V. Suspected conversion disorder: Foreseeable risks and avoidable errors. Acad Emerg Med 7(11): 1272-7, 2000.

Guggenheim, F.; Smith, G. Somatoform disorders. In: Kaplan, H.; Sadock, B. (eds.). Comprehensive textbook of psychiatry/VI. Wiliams \& Wilkins, Baltimore, pp. 1251-1279, 1995.

Legatt, A. D.; Rubin, M. J.; Kaplan, L. R.; Healton, E. B.; Brust, J. C. Global aphasia without hemiparesis: multiple etiologies. Neurology 37(2): 201-5, 1987.

Leo, R. J.; Konakanchi, R. Psychogenic respiratory distress: a case of paradoxical vocal cord dysfunction and literature review. Prim Care Companion. J Clin Psychiatry 1(2): 39-46, 1999.

Leslie, S. A. Diagnosis and treatment of hysterical conversion reactions. Arch Dis Child 63(5): 506-11, 1988.

Levy, R. S.; Jankovic, J. Placebo-induced conversion reaction: a neurobehavioral and EEG study of hysterical aphasia, seizure, and coma. J Abnorm Psychol 92(2): 243-9, 1983.

Lishman, W. Cerebrovascular disorders. Organic Psychiatry. The psychological consequences of cerebral disorder. Oxford, Blackwell Scientific Publications, pp. 21-78, 1987.

Lishman, W. A. Organic psychiatry: The psychological consequences of cerebral disorder. Oxford, Blackwell Science, p. 922, 1998.

Mace, C. J.; Trimble, M. R. Ten-year prognosis of conversion disorder. Br J Psychiatry 169(3): 282-8, 1996.

Ophir, D.; Katz, Y.; Tavori, I.; Aladjem, M. Functional Upper airway obstruction in adolescents. Arch Otolaryngol Head Neck Surg 116(10): 1208-9, 1990.

Roach, E. S.; Sparagana, S. P. Diagnosis of tuberous sclerosis complex. $J$ Child Neurol 19(9): 643-9, 2004.

Roelofs, K.; Keijsers, G. P.; Hoogduin, K. A.; Naring, G. W.; Moene, F. C. Childhood abuse in patients with conversion disorder. Am J Psychiatry 159(11): 1908-13, 2002.

Slater, E. T.; Glithero, E. A follow-up of patients diagnosed as suffering from "hysteria". J Psychosom Res 9(1): 9-13, 1965.

Smaira, S.; Kerr-Corrêa, F.; Contel, J. Psychiatric disorders and psychiatric consultation in a general hospital: a case-control study. Rev Bras Psiquiatr 25(1): 18-25, 2003

Steinhart, M. J. Conversion hysteria. Am Fam Physician 21(3): 125-9, 1980.

Stone, J.; Zeman, A.; Sharpe, M. Functional weakness and sensory disturbance. J Neurol Neurosurg Psychiatry 73(3): 241-5, 2002.

Stone, J.; Smyth, R.; Carson, A.; Lewis, S.; Prescott, R.; Warlow, C.; Sharpe, $M$. Systematic review of misdiagnosis of conversion symptoms and "hysteria". BMJ 331(7523): 989, 2005.

Stone, J.; Smyth, R.; Carson, A.; Warlow, C.; Sharpe, M. La belle indifference in conversion symptoms and hysteria: systematic review. $\mathrm{Br} J$ Psychiatry 188(3): 204-9, 2006.

Stoudemire, A.; Stork, M.; Simel, D.; Houpt, J. L. Neuro-ophthalmic systemic lupus erythematosis misdiagnosed as hysterical blindness. Am J Psychiatry 139(9): 1194-6, 1982.

Teasell, R. W.; Shapiro, A. P. Misdiagnosis of conversion disorders. Am J Phys Med Rehabil 81(3): 236-40, 2002.

Tranel, D.; Biller, J.; Damasio, H.; Adams Jr, H. P.; Cornell, S. H. Global aphasia without hemiparesis. Arch Neurol 44(3): 304-8, 1987.

Trojano, M.; Paolicelli, D. The differential diagnosis of multiple sclerosis: classification and clinical features of relapsing and progressive neurological syndromes. Neurol Sci 22(2): 98-102, 2001. 